\title{
Apego, cortisol y estrés en adultos, una revisión narrativa.
}

Attachment, cortisol and stress in adults, a narrative review.

\author{
Eduardo Agustín Reguera Nieto ${ }^{\text {a }}$. \\ ${ }^{a}$ Médico Adjunto de Psiquiatría. Hospital Universitario La Paz. Madrid, España. \\ Correspondencia: Eduardo Agustín Reguera Nieto (e.reguera.nieto@gmail.com)
}

Recibido: 27/12/2013; aceptado: 06/04/2014

\begin{abstract}
RESUMEN: En este artículo se presenta una revisión narrativa sobre la fisiopatología de los estados de insuficiente señalización glucocorticoidea en adultos y su relación con el estrés crónico. Estos serían comunes a toda una serie de trastornos interrelacionados, como el trastorno por estrés postraumático, el síndrome de fatiga crónica o la fibromialgia. En este sentido, se destaca la importancia de los estilos de apego inseguros como generadores/perpetuadores de dichos estados de hipocortisolismo, incluso en ausencia de sintomatología psiquiátrica. Hay cada vez mayores evidencias acerca de la posibilidad de revertir tal disfunción con técnicas psicoterapéuticas o farmacológicas. No obstante, se requiere mayor investigación sobre el impacto biológico de técnicas basadas en los modelos de apego y de su papel en los procesos de resiliencia.

PALABRAS CLAVE: apego a objetos, adultos, resiliencia, estrés, glucocorticoides, mineralocorticoides, sistema hipotálamo-hipofisario, sistema hipófiso-suprarrenal, psicoterapia, tratamiento.
\end{abstract}

\begin{abstract}
This article provides a narrative review of the pathophysiology of insufficient glucocorticoid signaling states in adults and their relation to chronic stress. These would be common to a number of interrelated disorders, such as posttraumatic stress disorder, chronic fatigue syndrome or fibromyalgia. In this sense, it highlights the importance of insecure attachment styles as generators / perpetuators of these states hypocortisolism, even in the absence of psychiatric symptoms. There is growing evidence about the possibility of reversing this dysfunction with psychotherapeutic or pharmacological techniques. However, further research on the biological impact of attachment-based techniques and their role in resilience processes is required.

KEY WORDS: object attachment, adults, resilience, stress, glucocorticoids, mineralocorticoids, hypothalamo-hypophyseal system, pituitary-adrenal system, psychotherapy, treatment.
\end{abstract}

\section{Introducción}

Tal y como se expuso en una revisión anterior sobre el mismo tema en infantes, ha aparecido una énfasis creciente en la literatura especializada en la relación entre estados de desregulación del eje hipotálamo - hipófisis - glándulas suprarrenales (eje HHA), hipocortisolismo funcional, situaciones traumáticas infantiles (1-2) y fallas graves en los vínculos primarios que se perpetuarían y cristalizarían en determinados estilos de apego. Respecto de las consecuencias fisiopatológicas de un estado de hipocortisolismo mantenido sí que hay una amplia unanimidad entre investigadores (3-4). En cuanto a las causas o mecanismos que justifican tal estado, parece haber un origen más complejo.

En la presente revisión se parte del substrato neurobiológico del estrés así como del modelo homeostasis - alostasis, descritos en el anterior trabajo. Se explora 
la compleja fisiopatología de los estados de insuficiente señalización glucocorticoidea en el adulto, su relevancia en la salud mental y la relación de los mismos con estilos de apego inseguros. Asimismo, el trabajo tratará de dar cuenta del estado del conocimiento actual sobre el tratamiento psicoterapéutico - farmacológico de los trastornos relacionados con el estrés crónico y de la relación entre apego y procesos de resiliencia.

\section{Metodología}

El objetivo de este trabajo es realizar una revisión narrativa sobre la relación entre los estados de insuficiente señalización glucocorticoidea del adulto, los trastornos psiquiátricos mediados por el estrés crónico y los estilos de apego. Asimismo, se revisan los tratamientos psicoterapéuticos o farmacológicos de tales estados de hipocortisolismo y la relación entre los estilos de apego y los procesos de resiliencia. Para realizar dicha revisión se realizó una búsqueda en Pubmed y Biblioteca Virtual en Salud mediante la combinación de los descriptores (incluidos en Medical Heading Subjects, MeSH) :" object attachment", "adults", "resilience", "stress", "glucocorticoids", "mineralocorticoids", "hypothalamo-hypophyseal system", "pituitary-adrenal system", "psychotherapy", "treatment" tanto en inglés como español entre los años 1986 y 2013, que se acompañó de una posterior búsqueda manual. Un total de 153 referencias fueron incluidas para su revisión, entre las que se pueden destacar 1 metaanálisis, 1 revisión sistemática, 14 ensayos clínicos aleatorizados, 21 estudios cuasi-experimentales, 18 estudios observacionales transversales, 11 estudios prospectivos de cohortes, 17 estudio de casos y controles, 9 estudios de serie de casos y 1 estudio de caso único. Además se incluyeron independientemente de su año de publicación artículos generadores de hipótesis, artículos de revisión y capítulos de libros.

\section{Los estados de hipocortisolismo y su fisiopatología}

Se podría definir la insuficiente señalización glucocorticoidea como cualquier estado en el que esté disminuida la capacidad de dichas hormonas de regular los sistemas relevantes implicados en la respuesta a estrés. El resultado final es una incapacidad del organismo para para responder al medio ambiente externo o interno a través de los glucocorticoides. Como veremos, esta desregulación del eje hipotálamo - hipófisis - corteza adrenal (eje HHA), se origina a múltiples niveles (3-5). 
El estado de hipocortisolismo podría aparecer así por una reducida biodisponibilidad hormonal a cualquiera de los niveles del eje HHA. En el espectro de trastornos relacionados con el estrés crónico hay evidencias de que podría tener lugar una insuficiencia adrenal primaria, una menor secreción de cortisol $(3,6)$. Apoya esta hipótesis el hecho de que en los estado de estrés crónico se han encontrado frecuentemente bajos niveles de cortisol, así como una respuesta pobre de cortisol a la administración de ACTH o CRH (4), lo cual ha sido comprobado en animales de experimentación (7). A ello puede contribuir la observada hipersecreción de CRF hipotalámico y la regulación a la baja de los receptores para CRF en la hipófisis (4, 6), con la consiguiente reducción en la biosíntesis hipofisaria de ACTH. A nivel de la corteza adrenal, uno de los agentes que podría estar implicado en la génesis de la disminución en la secreción de glucocorticoides sería el factor de transcripción ARNTL, el cual se ha comprobado como modulador del ritmo circadiano y la respuesta a estrés en roedores $(8)$.

Cada vez ha sido más conocida la implicación de la expresión de receptores glucocorticoideos en la desregulación del eje HHA, ya que dicho receptor es capaz de inducir su propia síntesis en la hipófisis (9). De este modo, en un funcionamiento estable del eje la concentración de receptores glucocorticoideos sería baja y el sistema seguiría una cinética lineal. Tras una situación de estrés mantenido en el tiempo parece que los receptores se mantendrían elevados de forma continuada, el sistema se empezaría a regir por cinéticas no lineales inestables y se acaba produciendo una inhibición mantenida de la secreción de ACTH en la hipófisis debido a la mayor concentración de complejos cortisol-receptor (10). Incluso se ha llegado a comprobar que experiencias repetidas de maltrato o adversidad infantil generan modificaciones epigenéticas del receptor glucocorticoideo secundarias a un incremento de la metilación de la región promotora del gen correspondiente (11). La tasa de incremento de metilación se correlacionaba inversamente de forma significativa con la reactividad de dichos receptores en el test de estimulación con CRH/Dexametasona (12), con la consiguiente desregulación del eje HHA. La metilación de dicho gen que codifica el receptor glucocorticoideo se ha demostrado relacionada igualmente con el humor deprimido materno durante la gestación, lo cual podría constituir un factor predisponente a nivel prenatal (13). Otros trabajos enfatizan la importancia que tiene el estrés infantil temprano sobre la regulación de la expresión de receptores glucocorticoideos a nivel de hipocampo y corteza frontal, lugares preferentes donde tiene el lugar el feed-back negativo sobre la síntesis de CRH y arginina-vasopresina (14). A través de todos estos mecanismos que actúan sobre los receptores glucocorticoideos, estaríamos hablando de un aumento de la sensibilidad de feed - back negativo del eje HHA (4).

Por otro lado, en los últimos años se ha explorado el papel de los receptores mineralocorticoideos para cortisol, especialmente presentes en el cerebro límbico y corteza prefrontal $(4,15-17)$. Dichos receptores (con una afinidad en el hipocampo 
por el cortisol 10 veces superior a los glucocorticoideos) se encargarían de mantener la actividad basal del eje HHA y el umbral de respuesta frente a estrés. De este modo, cuando tiene lugar el evento estresante, los receptores mineralcorticoideos se saturan rápidamente y el cortisol se une a los glucocorticoideos, ampliamente distribuidos por todo el cerebro (18). De esta forma, los estados de insuficiente señalización corticoidea podrían estar relacionados con las diferentes tasas de expresión de dichos receptores mineralocorticoideos y el desbalance consecuente en el ratio receptores MR/GR (15-17), con consecuencias notables en la regulación de la transcripción genómica (19). Aparecería así un desequilibrio entre los componentes activadores de los glucocorticoides y supresores de los mineralocorticoides en relación a la situación estresante (20-21). A pesar de que este balance está en buena medida genéticamente programado, es susceptible de sufrir desestabilizaciones en relación con fallas en la relación temprana con figuras significativas $(18,22)$. En este sentido, un trabajo encontró una asociación entre situaciones traumáticas infantiles de abandono o negligencia y una mayor reactividad de la amígdala frente a eventos estresantes, asociación que era modulada por los diferentes haplotipos del receptor mineralocorticoideo iso/val rs5522 (23).

Los receptores MR tienen un papel clave así en el mantenimiento de la homeostasis y en la regulación del umbral de disparo frente a la situación estresante (15-16, 21). Todo ello tendría lugar preferentemente en el núcleo paraventricular hipotalámico, donde se da la interacción de este sistema MR con el GR $(15,18)$. De ahí que los receptores MR sean esenciales para la comprensión de los mecanismos que subyacen al establecimiento de nuevos umbrales de disparo frente al estrés y al aumento del feed-back negativo del eje HHA $(15,21,24)$. De hecho, en un ensayo clínico aleatorizado en el que suministraba espironolactona (antagonista de los receptores MR) versus placebo a sujetos a los que posteriormente se les sometía a un test de estrés se comprobó un aumento de la biodisponibilidad del cortisol y una potenciación de los mecanismos de respuesta a estrés dependientes de los receptores GR (15) frente al grupo control, lo que demostraba los efectos moduladores de los receptores MR en la respuesta a eventos estresantes. Dicho bloqueo MR por espironolactona y el subsecuente desbalance MR/GR en la respuesta a estrés ha podido ser observado igualmente en animales de experimentación (25). Los receptores MR y sus posibles polimorfismos se han ido asentando en los últimos años como uno de los factores más decisivos, en su interacción con el cuidado parental, en la conformación de un estilo de apego seguro y una adecuada capacidad de autorregulación emocional (26). Parece cada vez más sólido el papel del factor neurotrófico derivado del cerebro (BDNF) en los mecanismos adaptativos frente al estrés crónico y depresión (27). En ratas de laboratorio sometidas a estrés crónico en laboratorio, se constató un incremento de BDNF en hipocampo, hipotálamo e hipófisis (28). Del mismo modo que en otro estudio se objetivó una elevación prolongada de la secreción de corti- 
ORIGINALES Y REVISIONES

coesteroides en ratas a las que experimentalmente se indujo una menor expresión de BDNF en hipocampo, lo cual apoya la hipótesis de este factor como otro regulador del eje HHA (29). Al igual que en el caso de los receptores corticoideos, también se han constatado cambios epigenéticos en la metilación del gen BDNF secundarios a situaciones traumáticas en la infancia temprana, lo cual alteraría de este modo la tasa de expresión de BDNF a nivel de la corteza prefrontal del adulto (30). Según un estudio reciente, esto posiblemente se dé a través de la modulación que ejerce el CRF hipotalámico sobre el crecimiento de espinas dendríticas inducido por BDNF (31).

Aparte de estos mencionados procesos de sensibilización, podría tener lugar una exposición crónica al cortisol, la cual generase daños neurológicos por estrés oxidativo que contribuyesen a esta disregulación crónica del eje HHA (32), por ejemplo, a través de un aumento de la histona deacetilasa (HDAC) (33). Esto ha sido especialmente demostrado a nivel del hipocampo, $(3,5,34-35)$ o a nivel del circuito amígdala-corteza prefrontal ventromedial (36-37). En uno de los estudios más recientes se objetivó igualmente una reducción de la sustancia gris a nivel del giro cingulado derecho en adultos con historia personal de trauma infantil, reducción que además era proporcional a la desregulación del cortisol tras el despertar (38). Otros investigadores evidenciaron una mayor activación del área límbica amígdala-hipocampo tras un test de estrés de laboratorio. Fue muy significativo en este trabajo experimental que los que presentaban la mayor activación de dicha área era los que presentaban menores elevaciones del cortisol tras el evento estresante (39). En este sentido, es especialmente revelador un estudio que se llevó a cabo sobre la densidad celular en hipocampo en pacientes con apegos inseguros. La hipótesis de la neurotoxicidad parece confirmarse, al menos parcialmente, por cuanto se objetivó una reducción bilateral de la densidad de ambos hipocampos para los pacientes con apego evitativo, mientras que en los que presentaban un apego ansioso se encontró una disminución significativa de la densidad del hipocampo izquierdo (40), lo cual provee una importante base neurobiológica para la comprensión de la regulación emocional en los estilos de apego inseguros.

Por último, como una causa complementaria aunque no suficiente para la desregulación humoral crónica también se ha hipotetizado una posible contribución por parte de un estado de disociación funcional de ambos hemisferios cerebrales derivado del trauma psicológico en la edad infantil (41). El resultado de todos estos procesos sería una desconexión franca entre los mecanismos de percepción del estrés actual y las reacciones naturales defensivas del cuerpo (42). 
Tabla 1.

Principales mecanismos implicados en el mantenimiento de los estados de insuficiente señalización glucocorticoidea.

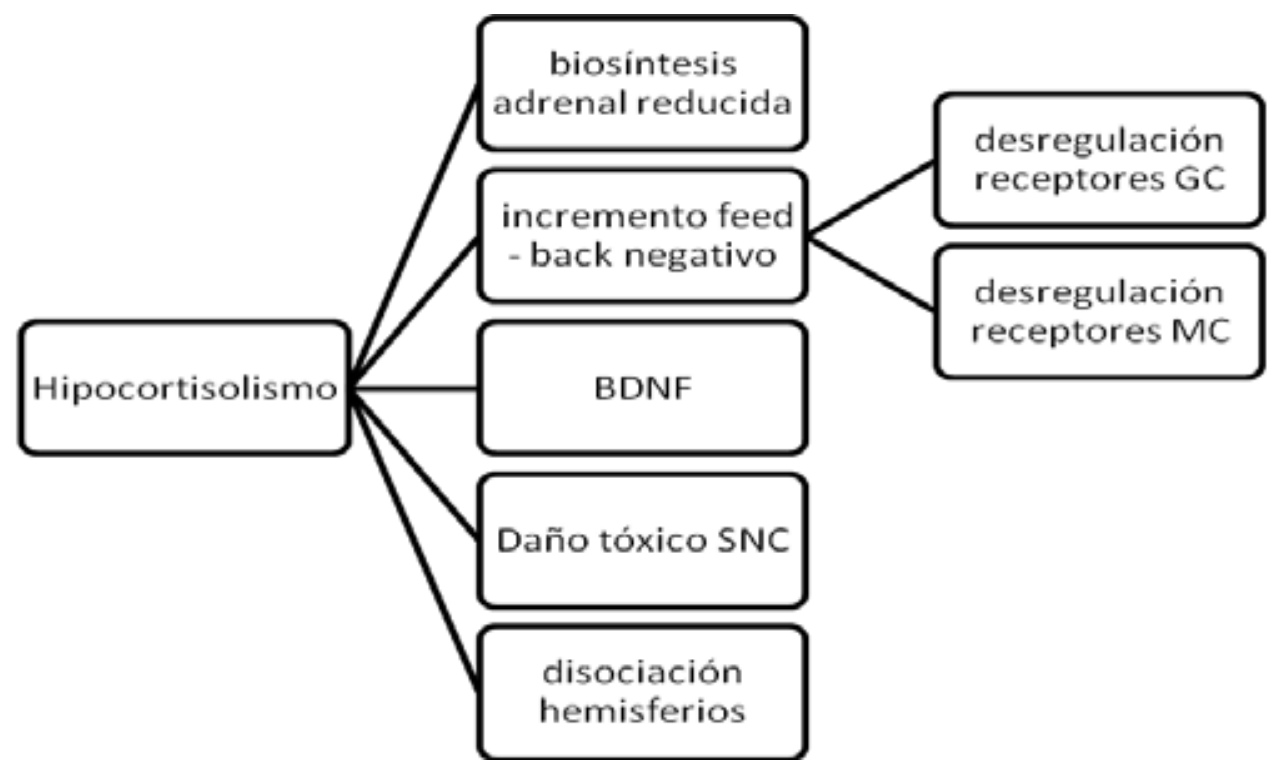

En resumen, los estados de insuficiente señalización glucocorticoidea parecen tener un origen muy complejo, en el que interactúan modificaciones en la tasa de expresión de receptores gluco y mineralocorticoideos, el papel coadyuvante del BDNF o las evidencias de neurotoxicidad directa. Ha habido autores como Dienstbier o Murray-Close que han abogado por el hipocortisolismo como evento adaptativo frente a situaciones de estrés crónico o de agresividad relacional, lo cual permitiría supuestamente una respuesta óptima frente al estrés (43), o bien, un aumento de la tolerancia frente al miedo (44) o las consecuencias negativas de los actos propios (45) o bien el establecimiento de una especie de alexitimia adaptativa (46). Incluso ciertos autores enfocan la cuestión desde un paradigma evolucionista, de modo que los estados de insuficiente señalización glucocorticoidea constituirían un modo en el que organismos sometidos por otros más dominantes y que podrían sufrir peores heridas en combate, disminuyen la respuesta inflamatoria dependiente del cortisol y potencian la respuesta inmune no específica $(3,47)$. En general, se asume que estos posibles efectos adaptativos tendrían lugar especialmente en los primeros momentos en los que aparecen los eventos estresantes, adaptación que se iría perdiendo a medida que se estereotipa la respuesta del eje HHA (4).

No obstante, parece cada vez más clara en la literatura especializada la consideración de dicho estado de hipocortisolismo como un evento alostático maladaptativo 
ORIGINALES Y REVISIONES

(48-51) (carga alostática) (52), que tendría lugar tras un estadio de hipercortisolismo adaptativo $(3,4,53-56)$. Estaríamos hablando de esta forma del correlato biológico del concepto psiquiátrico - psicoanalítico del trauma cumulativo (57). Entre estas podrían destacarse los mencionados cambios volumétricos en el cerebro, resistencia a la insulina y predisposición a la diabetes, osteoporosis, Asimismo, debido a los importantísimos efectos del cortisol en la modulación de la respuesta inflamatoria y de la inmunidad celular, estaría aumentada la vulnerabilidad para desarrollar trastornos autoinmunes, inflamatorios, síndrome de dolor crónico, alergias, endometriosis y asma (3-4, 54, 58-59). En los últimos años se ha estudiado asimismo cada vez más la asociación entre los estados de desregulación del eje HHA, síntomas de estrés y obesidad, en torno a la relación del cortisol y la grelina (60-61).

\section{Relevancia de los estados de hipocortisolismo crónico en el adulto}

En la literatura especializada, se está acumulando en los últimos años una notable evidencia sobre los estados de hipocortisolismo y la desregulación del eje HHA en relación con eventos estresantes infantiles. La teoría del apego y los diferentes estilos cognitivo - afectivos que de ella se postulan podrían representar una gran ayuda en la comprensión de los procesos psicobiológicos que subyacen a dichos estados de hipocortisolismo (62-64) en tanto el apego aparece como constructo imprescindible a la hora de ligar psicología del desarrollo, psiquiatría, psicoanálisis, neurobiología evolutiva y genética (65-68). Según algunos reputados investigadores de esta área, solamente la comunicación entre disciplinas vecinas podrá dar la clave para la comprensión de los trastornos de la fisiología del estrés y su relación con la psicología del desarrollo (69).

El hipocortisolismo contrarregulatorio aparecería como un mecanismo central en la comprensión de diferentes trastornos relacionados con un estrés crónico, tales como el trastorno por estrés postraumático, fibromialgia, síndrome de fatiga crónica, síndrome de intestino irritable, depresiones atípicas, síndrome de burn-out, trastornos de ansiedad, e incluso amenorrea hipotalámica funcional o síndrome de intestino irritable $(1,3-4,56,64,70-78)$. Algunos autores llegan a considerar el hipocortisolismo como un factor de riesgo para desarrollar una serie de trastornos psicosomáticos como los anteriores y su clínica correspondiente (79). En el caso de la fibromialgia, dicha correlación llega a ser notable en tanto ciertos autores han descrito asociaciones entre la gravedad del hipocortisolismo y la intensidad del dolor (80-81) o una peor respuesta al tratamiento psicoterapéutico en el síndrome de fatiga crónica (82). La hipótesis del hipocortisolismo ha podido ser confirmada ampliamente en experimentos con otros mamíferos sometidos a pruebas de inoculación de estrés (83- 85), incluso tras una sola exposición a un estresor severo (86). 
En humanos, la relación entre los mencionados estados de hipocortisolismo y síntomas de estrés crónico ha sido ampliamente documentada. En un estudio observacional, se evidenció cómo los vecinos de un barrio con altos niveles de estrés y bajos niveles de soporte social presentaban bajas cifras de cortisol basal, así como tras un estresor significativo (53). Desde otro ángulo diferente, otro trabajo constató una respuesta aplanada de cortisol en pacientes homosexuales que habían crecido en ambientes marcadamente estigmatizantes y estresantes, en comparación con otros pacientes homosexuales que lo habían hecho en ambientes más tolerantes (87). Incluso se ha llegado a constatar en jóvenes adultos griegos a los que se les analizaba el cortisol capilar una asociación entre la regulación a la baja del eje HHA, síntomas de estrés y acontecimientos vitales adversos, en comparación con un grupo control de jóvenes adultos suecos (55). Un trabajo de investigación muy interesante evaluó a los psicoterapeutas en formación que atendían a pacientes con trastorno límite de personalidad y que habían protagonizado recientemente intentos de suicidio, lo que constituye según los autores un potente estresor de carácter interpersonal para los psicoterapeutas. Encontraron que, previamente a sesiones que los psicoterapeutas preveían que iban a ser complicadas, aparecía una disociación entre SNA y el eje HHA, de tal forma que la alfa-amilasa podía aparecer elevada y el cortisol incluso suprimido. Estos parámetros se normalizaban posteriormente cuando la sesión transcurría bien, pero no sucedía cuando el final de la misma no era satisfactorio, por nuevas amenazas de suicidio por ejemplo (88).

Los diferentes estilos de apego inseguro perpetuarían según esta línea de pensamiento una situación de estrés crónico más o menos pronunciada en relación con la gravedad del daño vincular y la incapacidad de recurrir a mecanismos de defensa del yo más avanzados. Las relaciones interpersonales de convertirían de este modo en una fuente constante de estrés. Esto daría lugar al estado referido de hipocortisolismo, así como a una disociación del eje HHA frente al simpático adrenal - adrenalina/ noradrenalina - , en la que el segundo presenta una elevación sostenida $(41,89)$. Se ha llegado a comprobar dicha desregulación del eje HHA incluso en personas que fueron expuestas a situaciones traumáticas infantiles pero que no desarrollaron morbilidad psiquiátrica, lo cual parece apoyar esta hipótesis $(2,72,90)$.

De este modo, en un estudio se exploró la relación entre el cortisol salivar, el estilo de apego y las situaciones estresantes provocadas en laboratorio. Los investigadores comprobaron que la menor concentración de cortisol correspondía al grupo de apego temeroso, seguido por el grupo preocupado y por último, el seguro (62). En otro trabajo se evaluaba el cuidado materno autopercibido por el sujeto en etapas tempranas y se intentaba relacionar con la respuesta del cortisol al estrés. Dividieron a la muestra en tres grupos: bajo, medio y alto cuidado materno percibido. En contra de lo esperado por el grupo investigador, encontraron que tanto el grupo de bajo como de alto cuidado mostraron una curva aplanada o reducida de respuesta del cor- 
tisol al estrés, mientras que en el grupo de cuidado intermedio evidenciaron una alta respuesta del cortisol. En su discusión, argumentan la hipótesis del hipocortisolismo como una situación de adaptación crónica y regulación a la baja del eje HHA (91). Resultados similares fueron obtenidos en otro estudio, en el que se exponía a un estresor artificial psicosocial a hombres y mujeres. Encontraron una respuesta aplanada del cortisol en sujetos con antecedentes de eventos adversos en la infancia, especialmente varones, mientras que no se observaron diferencias en las cifras basales (92). Particularmente interesante es otro trabajo muy reciente en el que se estudiaba a adultos jóvenes, su ajuste psicosocial, sus niveles de cortisol y cómo evaluaban posibles conflictos de sus propios padres. Se encontró que los sujetos procedentes de familias con escasos ingresos tendían a tener peores valoraciones de los conflictos parentales y un peor ajuste psicosocial, sin que esto se relacionase con alteraciones del cortisol. En cambio, se objetivó que aquellos sujetos que tendían a sentirse responsables de los conflictos de sus padres mostraban cifras de cortisol disminuidas en la mañana y una curva de secreción aplanada a lo largo del día, en comparación con aquellos que no se culpaban por los conflictos de relación de sus padres (93). Se puede deducir así en el grupo que presenta hipocortisolismo alteraciones en los procesos de especularidad (mirroring) parental y por tanto, en los modelos de apego. Incluso se ha podido demostrar experimentalmente en un gran estudio prospectivo presentado recientemente cómo los bebés que habían tenido escasa interacción con sus madres a la edad de 3 meses presentaban una hiporreactividad del eje HHA a los 19 años cuando eran sometidos a situaciones estresantes en laboratorio (94). Curiosamente, estos hallazgos sólo se encontraban en varones. En otro estudio trataron de demostrar que un fallecimiento de un progenitor a edad temprana junto con una pobre calidad de las relaciones familiares podía incrementar a largo plazo el riesgo cardiovascular y la reactividad del cortisol al estrés. Esperaban la mayor elevación del cortisol en adultos que cumplían ambos factores, pero encontraron que la mayor elevación correspondía a los que no los presentaban, optando asimismo por la hipótesis del hipocortisolismo (95). Un trabajo exploró la relación entre los ritmos circadianos de cortisol y la agresividad física y relacional. Mientas que la física se relacionaba con elevaciones de cortisol y rápidos descensos, la agresividad relacional se relacionaba con una cifra de cortisol matutino reducida así como una curva suprimida a lo largo del día (45).

En ciertos estudios, las alteraciones del eje HHA no aparecían espontáneamente en las analíticas sino tras pruebas de estimulación. En esta línea, uno de los trabajos estudió a sujetos con antecedentes de exposición a eventos traumáticos en la infancia que no habían desarrollado sintomatología psiquiátrica posteriormente con un grupo de sujetos sin patología y sin antecedentes traumáticos en la infancia. Constataron en el primer grupo una respuesta atenuada del cortisol tras la estimulación con CRH/ dexametasona pero no encontraron diferencias en el cortisol salivar, marcador del funcionamiento basal del eje HHA (2). Igualmente, en otro trabajo se evidenció una 
correlación entre los niveles de ACTH post-estimulación con CRH y el grado de estrés familiar y unión parental, mientras que no se encontraron alteraciones basales del eje HHA (96). Un estudio evidenció nuevamente una respuesta corticoidea suprimida tras el test de estimulación en relación con situaciones de abuso emocional infantil, que se potenciaba con la edad y era independiente de diagnósticos psiquiátricos y puntuaciones sintomáticas (97). De modo congruente con el estudio anterior, en otro se pudo objetivar una correlación negativa entre situaciones de sobreprotección parental durante la niñez o adolescencia en una serie de adultos, hipoactividad del eje HHA en el test de estimulación con dexametasona y reducción del volumen del hipocampo (35). Otro estudio encontró una relación entre una historia de abuso sexual en mujeres sin cuadros depresivos en la actualidad y una sensibilización de la hipófisis anterior tras la administración de CRH y ACTH junto con mecanismos regulatorios adaptativos a nivel de la corteza adrenal (6).

Resumiendo, los estados de insuficiente señalización glucocorticoidea son cada vez más aceptados como una consecuencia psicofisiológica de toda una serie de trastornos relacionados con situaciones de estrés mantenido. En los últimos años es cuando ha sido cada vez más explorado el posible papel de las situaciones traumáticas infantiles y los estilos de apego inseguros como generadores de estrés crónicos en las relaciones interpersonales, incluso en ausencia de morbilidad psiquiátrica franca. Es por ello, que estilo de apego y los modelos internos de trabajo asociados aparecen como constructos decisivos a la hora de aportar comprensión y posibilidades terapéuticas en todo este espectro de patologías.

\section{Reversibilidad y tratamiento de los estados de desregulación glucocorticoidea}

Como se observa a través de las diversas investigaciones referidas, la literatura psiquiátrica ha ido acumulando evidencia en los últimos años sobre los mencionados estados de hipocortisolismo secundarios a situaciones de estrés crónico, tal como puedan ser las perpetuadas por los diferentes estilos de apego inseguro. En infantes, un notable número de autores ha demostrado la capacidad elástica de los sistemas biológicos adaptativos, de tal forma que los procesos psicoterapéuticos o modificaciones en los vínculos con las figuras significativas pueden generar una normalización de la respuesta humoral frente al estrés. En adultos, los estudios y las conclusiones son más débiles, de tal forma que hay muy escasos trabajos de investigación que exploren la posible reversibilidad de los estados de insuficiente señalización glucocorticoidea.

Sí que aparecen varios estudios sobre técnicas psicoterapéuticas que han demostrado reducir las cifras de cortisol en sujetos con situaciones de hiperreactividad corticoidea; es decir, previamente a la regulación a la baja del eje HHA. Muchos 
ORIGINALES Y REVISIONES

de ellos se basan en técnicas de meditación, como el entrenamiento basado en mindfulness o atención plena (98-100), otros en técnicas psicoterapéuticas que buscan incrementar la competencia o plasticidad emocional (101), o a través de sesiones de musicoterapia en pacientes que van a ser sometidos a procedimientos invasivos (102) o que presentaban cánceres terminales (103), o bien gracias a técnicas de meditación (104-105). En otro estudio digno de mención se realizó un ensayo clínico aleatorizado en el que asignaba a mujeres con amenorrea hipotalámica funcional a recibir una psicoterapia cognitivo - conductual frente a un grupo control que solamente recibía observación. Las que recibieron psicoterapia no solamente reducían las cifras de cortisol sino que también restauraba la función ovárica, frente al grupo de observación, en el cual no tenía lugar ninguno de estos cambios (77). Otros investigadores demostraron cómo una psicoterapia cognitivo - conductual centrada en el manejo del estrés contribuía a disminuir la hiperreactividad glucocorticoidea en pacientes con dermatitis atópica frente al grupo control (106).

Respecto a las situaciones de hipocortisolismo contrarregulatorio los estudios de investigación son escasos y dispersos. En este caso no se ha logrado encontrar trabajos que exploren específicamente la posible reversibilidad de esta desregulación humoral secundaria a estilos de apego inseguros. No obstante, ciertos autores que trabajan con psicoterapias psicodinámicas sí encuentran factible tal reversión al actuar de esta forma sobre las regiones cerebrales reguladoras (64-65, 107-108). Sí que aparecerían otros trabajos que constatan un cambio en otros estados de hiporreactividad corticoidea, por ejemplo, tras programas de yoga (109). Especialmente interesante en este sentido es un trabajo en el que se evaluó el impacto de un programa de 8 semanas de reducción de estrés basado en mindfulness sobre las cifras de cortisol salivar tras el despertar en pacientes que habían sido tratadas de un cáncer de mama. Los investigadores objetivaron un incremento significativo de los niveles de dicho parámetro en los test completados tras el entrenamiento. Al comparar sus datos con los de otros estudios que evaluaban las técnicas de mindfulness, concluían que las pacientes partían de cifras aplanadas de cortisol secundarias a la exposición a experiencias estresantes asociadas con el diagnóstico y tratamiento del cáncer (110). Resultados similares se encontraron en un ensayo clínico posterior, en el que la psicoterapia basada en mindfulness aplicada sobre pacientes que habían sobrevivido a un cáncer de mama normalizaba las cifras de cortisol, en comparación con los supervivientes que no la recibían, las cuales presentaban curvas de secreción aplanadas (111). En otro estudio con el mismo tipo de pacientes se encontraron datos similares aplicando otro tipo de psicoterapia de grupo (112). Estos datos armonizarían de esta forma con los de otros investigadores, que observaron una disminución de las cifras de cortisol en pacientes con cáncer de mama en estadio inicial a las que se ofrecía psicoterapia cognitivo conductual para controlar los síntomas de estrés, un estadio hipotéticamente previo al anterior (113). Otros trabajos también constataron una reversibilidad de los estados 
de hiporreactividad en el caso de pacientes con síntomas de burn-out, que se correlacionaban con una mejoría sintomática (70), o en el caso de pacientes con trastorno por estrés postraumático, donde el incremento de los niveles de cortisol sólo se daba en pacientes no deprimidos (114). Igualmente, en otro trabajo en el que se trataba a pacientes con síndrome de fatiga crónica con psicoterapia cognitivo - conductual se encontró una reversión del estado de hipocortisolismo, que además era paralela a la mejoría clínica (115).

En cuanto a posibles tratamientos farmacológicos que pudieran paliar o revertir los mencionados estados de hipocortisolismo, hay una gran escasez de estudios. Sí que parece haber más evidencia de la mejoría de los trastornos por estrés postraumático con glucocorticoides exógenos (116-118) o incluso, de la posibilidad de prevenir síntomas de TEPT con tratamientos glucocorticoideos tras la exposición de un sujeto a un evento gravemente estresante (119-120). Asimismo, se han encontrado mejorías tanto analítica como sintomáticas con el tratamiento glucocorticoideo en pacientes con síndrome de fatiga crónica (121-122) y fibromialgia (123). A pesar del pequeño número de estudios relativos al tratamiento farmacológico con glucocorticoides, ciertos autores postulan que pueda ser una opción factible para el grupo de trastornos caracterizados por estados de hipocortisolismo (124).

En resumen, cada vez hay mayor evidencia acerca de la posibilidad de revertir los estados de insuficiente señalización glucocorticoidea con diferentes técnicas de psicoterapia, entre las cuales, han destacado en este sentido las basadas en mindfulness. A pesar de que los diferentes estilos de apego inseguro aparecen ya cada vez más claramente como factores de riesgo para desarrollar complicaciones somáticas derivadas un estrés mantenido, prácticamente no hay prácticamente estudios que evalúen con una metodología rigurosa la posible normalización del eje HHA, por ejemplo, a través de las psicoterapia basada en la mentalización. En cuanto a los tratamientos farmacológicos, sí que se ha habido evidencia en los últimos años de mejorías con glucocorticoides en diferentes patologías relacionadas con el estrés crónico, especialmente el TEPT, el síndrome de fatiga crónica y la fibromialgia. Se requerirían más trabajos de investigación para conocer si se puede extrapolar dicha mejoría a otros trastornos del mismo espectro.

\section{Apego y resiliencia}

La resiliencia se ha teorizado como un fenómeno que resulta de la integración de sistemas humanos adaptativos que cooperan exitosamente cuando se produce una exposición a situaciones estresantes, adversas o traumáticas. Lo que se posibilitaría así es una flexibilidad adaptativa que permita el salto desde experiencias pasadas 
negativas hacia la superación de las vicisitudes de la vida diaria (125). O bien, desde el modelo de estrés y alostasis, se podría definir la resiliencia como la habilidad de un organismo para responder a estresores en su entorno a través de un adecuado uso y de una eficiente finalización de las respuestas alostáticas (126). Desde su origen en la termodinámica como la capacidad de un ecosistema de regresar a la estabilidad después de una alteración (48), pasó al estudio de niños en situaciones en riesgo psicosocial y posteriormente, a manejarse como concepto clave en el estudio de los procesos que confieren protección frente al trauma (127), lo que ha permitido operativizarlo y diseñar intervenciones basadas en la resiliencia (128).

Ha sido recientemente cuando el apego ha sido postulado como un factor esencial en la comprensión de los procesos que median la resiliencia de los individuos frente a situaciones estresantes y potencialmente traumatizantes (129-131), así como frente a las situaciones de fatiga por compasión en profesionales (132). De esta forma, se conectan los procesos más biológicos de estrés con los factores más sociales - contextuales en el desarrollo del ser humano (133-136), lo cual resulta esencial para una comprensión holística de dichos trastornos (69). Nos ha permitido profundizar en el entendimiento de la regulación emocional, capacidad fundamental de la persona y elemento imprescindible para la consecución de la salud mental (137-139).

El apego seguro permite la internalización progresiva de la capacidad propia de regulación afectiva. Se produce así un entonamiento afectivo y fisiológico entre padres e hijos que permite a estos últimos modelar de forma cada vez más fina su sistema de respuesta a estrés (140). Un apego no seguro desregularía dicha capacidad al alza o a la baja. En este caso, lo perdido no sería el vínculo sino la posibilidad de establecer mecanismos regulatorios de alto nivel, lo cual parece permanecer en las relaciones posteriormente significativas (141-142). En la misma línea, estilos de apego inseguros dificultarían un manejo adaptativo de los síntomas de estrés en la infancia, paso clave para un correcto manejo en la edad adulta (143).

A un nivel más biológico, lo que este nuevo modelo explicativo pretende dar cuenta es del desarrollo parejo y en paralelo tanto del Sistema Nervioso Central como del self. No habría un correcto desarrollo de la arquitectura cerebral y de sus interconexiones sino a través de un adecuado equilibrio entre la calidad del ambiente físico, la del ambiente relacional y de los vínculos cuidadores (65), con repercusiones demostradas en la activación cerebral de ciertas regiones en función de la presencia o ausencia de ciertas experiencias tempranas (144). Todo ello converge con la llamada neurobiología interpersonal, teoría desarrollada por Daniel Siegel en torno al desarrollo interconectado de mente, cerebro y relaciones vinculares (145). Aparte de las notables implicaciones clínicas asociadas, este nuevo paradigma viene a dar una vuelta de tuerca más a la problemática que supone la causalidad en la Psiquiatría actual, especialmente la de tendencia más biológica - mecanicista. Si hay algo que dinamita a nivel neurobiológico la teoría del apego es la deformación de lo Real que 
introduce la dialéctica causa - consecuencia, problemática que radica en el núcleo mismo de la Psiquiatría como disciplina (146). Dicho sea de paso, el genial Nietzsche ya intuyó tal falsedad inherente a la diferenciación cartesiana entre res cogitans y res extensa, poniendo el acento no en el dualismo ontológico como tal - tesis de Damasio y otros - sino en el dualismo causalista (147).

De este modo, los avances en la comprensión de dicha reciprocidad somatopsíquica han permitido poner en relación la psicología del desarrollo con las neuronas espejo (148), los marcadores somáticos (149), el papel de la vía directa - sin intervención cortical - tálamo-amígdala en el miedo aprendido (150), la función reguladora orbito-frontal (151) o el giro cingulado posterior y su relación con mecanismos de defensa frente a situaciones emocionalmente perturbadoras (108). O echar luz sobre como la inoculación progresiva de situaciones de estrés en el infante - por ejemplo, a través separaciones intermitentes respecto de sus padres - induciría el desarrollo neural, por cuanto potencia el control cognitivo prefrontal de las conductas e incrementa la densidad del córtex a nivel frontal ventromedial (152). Asimismo, cada vez existen mayores evidencias del importante papel de la corteza prefrontal en los procesos de mentalización, así como las tareas integradoras - especialmente de estímulos visuales - del lóbulo temporal. De esta forma los procesos mentalizadores requerirían un control ejecutivo flexible y sujeto a un continuo feedback respecto a las emociones propias y a las inferidas de las figuras cuidadoras (153).

La teoría del apego, desde sus inicios psicoanalíticos, se ha consolidado progresivamente como una auténtica teoría-puente en el seno de la Psiquiatría, la Psicología y todas las disciplinas vecinas (66). Especialmente importante es su visión unificadora de lo biológico y lo psicológico en torno a las relaciones tempranas con las figuras significativas y sus repercusiones neurales. Asimismo, en los últimos años ha contribuido a potenciar el debate en torno a la resiliencia, a los mecanismos que la subyacen y a las posibilidades terapéuticas que permite.

\section{Conclusiones}

Los estados de insuficiente señalización glucocorticoidea se han ido destacando en los últimos años como procesos biológicos esenciales en la comprensión de toda una serie de patologías relacionadas con el estrés crónico. La génesis de dicho estado alostático es muy compleja, de tal forma que implica una desregulación de los receptores glucocorticoideos y mineralocorticoideos, una disminución de la síntesis de estas hormonas en la cápsula suprarrenal, una modulación del eje HHA por el BDNF y un daño directo del hipocampo y otros centros neurales, por citar algunos de los factores más relevantes y contrastados. 
El hipocortisolismo crónico ha sido cada vez más aceptado como una consecuencia psicofisiológica dañina para el organismo, aunque hay ciertos autores que postulan ciertas ventajas adaptativas, al menos en sus fases iniciales. El concepto de carga alostática da cuenta precisamente de esta potencial maladaptación del organismo y sus consecuencias. Sí que existe amplia unanimidad en torno a todos los trastornos médicos que pueden derivarse de un eje HHA frenado crónicamente y la desregulación endocrino-inmunitaria que ello conlleva, entre los que pueden destacarse los trastornos autoinmunes e inflamatorios, síndromes de dolor crónico, alergias, asma, etc.

La posibilidad de revertir los estados de insuficiente señalización glucocorticoidea ha ido consolidándose en estos últimos años como un foco de investigación y posible avance terapéutico. Mientras que los estados de hiperreactividad corticoidea y su normalización han sido ampliamente descritos, todavía existe una notable laguna en lo que respecta a los estados de hipocortisolismo. Se han descrito normalizaciones en este sentido con técnicas basadas en la meditación o en la atención plena, principalmente. Pese a la creciente importancia de los estilos de apego en su relación con el estrés crónico, existe todavía una marcada carencia de estudios que investiguen la posibilidad de revertir dichos estados con intervenciones técnicas específicas, del estilo de la psicoterapia basada en la mentalización. Respecto a los tratamientos farmacológicos, se han descrito mejorías sintomáticas y analíticas con glucocorticoides exógenos en un número muy limitado de estudios, sin que todavía sea posible trasladar estos resultados a todo el espectro de patologías relacionadas con el estrés crónico. Tanto en el ámbito de las psicoterapias como de la farmacología hormonal, hay una patente necesidad de mayor investigación en torno a la posibilidad de procurar mejorías a todas estos trastornos, frente a los cuales los recursos terapéuticos son escasos.

La teoría del apego, desde su origen psicoanalítico, se ha consolidado dentro del corpus teórico de la Psiquiatría como un nuevo paradigma en la comprensión del desarrollo humano así como de los correlatos psíquico - biológicos. En los últimos años ha habido un especial énfasis en demostrar cómo los daños a nivel vincular y representacional en edades tempranas pueden dar lugar a alteraciones prolongadas en los sistemas biológicos de respuesta a estrés, especialmente el eje hipocampo - hipotálamo - hipófisis - corteza adrenal, además de otras regiones encefálicas. La teoría del apego se ha ido consolidando como una auténtica teoría - puente entre disciplinas afines en el campo de la Salud Mental. Este carácter interdisciplinar es esencial a la hora de entender y tratar las patologías mencionadas, siempre a caballo entre lo biológico, lo psicológico y lo social. 


\section{BIBLIOGRAFÍA}

(1) Binder E, Holsboer F. Low cortisol and risk and resilience to stress-related psychiatric disorders. Biol Psychiatry. 2012; 71: p. 282-283.

(2) Klaassens E. Bouncing back - trauma and the HPA-axis in healthy adults. Eur J Psychotraumatol. 2010.

(3) Raison C, Miller A. When Not Enough Is Too Much: The Role of Insufficient Glucocorticoid Signaling in the Pathophysiology of Stress-Related Disorders. Am J Psychiatry. 2003; 160: p. 1554-1565.

(4) Heim C, Ehlert U, Hellhammer D. The potential role of hypocortisolims in the pathophysiology of stress-related bodily disorders.. 2000 Jan;25(1):1-35. Psychoneuroendocrinology. 2000 Jan; 25(1): p. 1-35.

(5) Sapolsky R, Krey L, McEwen B. The neuroendocrinology of stress and aging: the glucocorticoid cascade hypothesis. Endocr Rev. 1986; 7: p. 284-301.

(6) Heim C, Newport D, Bonsall R, Miller A, Nemeroff C. Altered Pituitary-Adrenal Axis Responses to Provocative Challenge Tests in Adult Survivors of Childhood Abuse. Am J Psychiatry. 2001; 158: p. 575-581.

(7) Saltzman W, Hogan B, Abbott D. Diminished cortisol levels in subordinate female marmosets are associated with altered central drive to the hypothalamic-pituitary-adrenal axis. Biol Psychiatry. 2006 Oct; 15;60(8): p. 843-9.

(8) Leliavski A, Shostak A, Husse J, Oster H. Impaired Glucocorticoid Production and Response to Stress in Arntl-Deficient Male Mice. Endocrinology. 2013 Nov 4.

(9) Gupta S, Aslakson E, Gurbaxani B, Vernon S. Inclusion of the glucocorticoid receptor in a hypothalamic pituitary adrenal axis model reveals bistability. Theor Biol Med Model. 2007 Feb; $14(4: 8)$.

(10) Gupta S, Aslakson E, Gurbaxani B, Vernon S. Inclusion of the glucocorticoid receptor in a hypothalamic pituitary adrenal axis model reveals bistability. 2007 Feb; 14;4:8.

(11) Reynolds R. Glucocorticoid excess and the developmental origins of disease: two decades of testing the hypothesis--2012 Curt Richter Award Winner. Psychoneuroendocrinology. 2013 Jan; 38(1)( doi: 10.1016/j.psyneuen.2012.08.012): p. 1-11.

(12) Tyrka A, Price L, Marsit C, Walters O, Carpenter L. Childhood Adversity and Epigenetic Modulation of theLeukocyte Glucocorticoid Receptor. PLoS ONE. 2012 Jan; 7(1).

(13) Oberlander T, Weinberg J, Papsdorf M, Grunau R, Misri S, Devlin A. Prenatal exposure to maternal depression, neonatal methylation of human glucocorticoid receptor gene (NR3C1) and infant cortisol stress responses. Epigenetics. 2008 Mar; 3(2): p. 97-106.

(14) Meaney M, Diorio J, Francis D, Widdowson J, LaPlante P, Caldji C, et al. Early environmental regulation of forebrain glucocorticoid receptor gene expression: implications for adrenocortical responses to stress. Dev Neurosci. 1996; 18(1-2): p. 49-72.

(15) Cornelisse S, Joëls M, Smeets T. A randomized trial on mineralocorticoid receptor blockade in men: effects on stress responses, selective attention, and memory. Neuropsychopharmacology. 2011 Dec; 36(13)(doi: 10.1038/npp.2011.162): p. 2720-8.

(16) Timmermans W, Xiong H, Hoogenraad C, Krugers H. Stress and excitatory synapses: from health to disease. Neuroscience. 2013 Sep; 17;248(doi: 10.1016/j.neuroscience.2013.05.043): p. 626-36. 
(17) Qi X, Kamphuis W, Wang S, Wang Q, Lucassen P, Zhou J, et al. Aberrant stress hormone receptor balance in the human prefrontal cortex and hypothalamic paraventricular nucleus of depressed patients. Psychoneuroendocrinology. 2013 Jun; 38(6)(doi: 10.1016/j.psyneuen.2012.09.014): p. 863-70.

(18) De Kloet E, Vreugdenhil E, Oitzl M, Joëls M. Brain corticosteroid receptor balance in health and disease. Endocr Rev. 1998 Jun; 19(3): p. 269-301.

(19) Zalachoras I, Houtman R, Meijer O. Understanding stress-effects in the brain via transcriptional signal transduction pathways. Neuroscience. 2013 Jul; 9;242(doi: 10.1016/j.neuroscience.2013.03.038): p. 97-109.

(20) De Kloet E. Stress: a neurobiological perspective. Tijdschr Psychiatr. 2009; 51(8): p. 541-50.

(21) Joëls M, Sarabdjitsingh R, Karst H. Unraveling the time domains of corticosteroid hormone influences on brain activity: rapid, slow, and chronic modes. Pharmacol Rev. 2012 Oct; 64(4) (doi: 10.1124/pr.112.005892): p. 901-38.

(22) De Kloet E. Functional profile of the binary brain corticosteroid receptor system: Mediating, multitasking, coordinating, integrating. Eur J Pharmacol. 2013 Nov; 5;719(1-3)(doi: 10.1016/j. ejphar.2013.04.053): p. 53-62.

(23) Bogdan R, Williamson D, Hariri A. Mineralocorticoid receptor Iso/Val (rs5522) genotype moderates the association between previous childhood emotional neglect and amygdala reactivity. Am J Psychiatry. 2012 May; 169(5)(doi: 10.1176/appi.ajp.2011.11060855): p. 515-22.

(24) Harris A, Holmes M, de Kloet E, Chapman K, Seck1 J. Mineralocorticoid and glucocorticoid receptor balance in control of HPA axis and behaviour. Psychoneuroendocrinology. 2013 May; 38(5)(doi: 10.1016/j.psyneuen.2012.08.007): p. 648-58.

(25) Ruiz-Aizpurua L, Buwalda B, De Boer S. Acute and lasting effects of single mineralocorticoid antagonism on offensive aggressive behaviour in rats. Behav Processes. 2013 Sep; 98( doi: 10.1016/j.beproc.2013.05.011): p. 72-7.

(26) Luijk M, Tharner A, Bakermans-Kranenburg M, van Ijzendoorn M, Jaddoe V, Hofman A, et al. The association between parenting and attachment security is moderated by a polymorphism in the mineralocorticoid receptor gene: evidence for differential susceptibility. Biol Psychol. 2011 Sep; 88(1)(doi: 10.1016/j.biopsycho.2011.06.005): p. 37-40.

(27) Kunugi H, Hori H, Adachi N, Numakawa T. Interface between hypothalamic-pituitaryadrenal axis and brain-derived neurotrophic factor in depression. Psychiatry Clin Neurosci. 2010 Oct; 64(5)( doi: 10.1111/j.1440-1819.2010.02135.x): p. 447-59.

(28) Naert G, Ixart G, Maurice T, Tapia-Arancibia L, Givalois L. Brain-derived neurotrophic factor and hypothalamic-pituitary-adrenal axis adaptation processes in a depressive-like state induced by chronic restraint stress. Mol Cell Neurosci. 2011 Jan; 46(1): p. 55-66.

(29) Taliaz D, Loya A, Gersner R, Haramati S, Chen A, Zangen A. Resilience to Chronic Stress Is Mediated by Hippocampal Brain-Derived Neurotrophic Factor. J Neurosci. 2011 Mar; 31(12): p. 4475-4483.

(30) Roth T, Lubin F, Funk A, Sweatt J. Lasting epigenetic influence of early-life adversity on the BDNF gene. Biol Psychiatry. 2009 May; 65(9): p. 760-769.

(31) Bennett M, Lagopoulos J. Stress and trauma: BDNF control of dendritic-spine formation and regression. Prog Neurobiol. 2013 Nov; pii: S0301-0082(13)00116-0(doi: 10.1016/j.pneurobio.2013.10.005). 
(32) Aschbacher K, O’Donovan A, Wolkowitz O, Dhabhar F, Su Y, Epel E. Good stress, bad stress and oxidative stress: insights from anticipatory cortisol reactivity. Psychoneuroendocrinology. 2013 Sep; 38(9)(doi: 10.1016/j.psyneuen.2013.02.004): p. 1698-708.

(33) Jason L, Sorenson M, Sebally K, Alkazemi D, Lerch A, Porter N, et al. Increased HDAC in association with decreased plasma cortisol in older adults with chronic fatigue syndrome. Brain Behav Immun. 2011 Nov; 25(8)(doi: 10.1016/j.bbi.2011.04.007): p. 1544-7.

(34) McEwen B, Seeman T. Protective and damaging effects of mediators of stress: elaborating and testing the concepts of allostasis and allostatic load. Ann N Y Acad Sci. 1999; 896: p. 30-47.

(35) Narita K, Fujihara K, Takei Y, Suda M, Aoyama Y, Uehara T, et al. Associations among parenting experiences during childhood and adolescence, hypothalamus-pituitary-adrenal axis hypoactivity, and hippocampal gray matter volume reduction in young adults. Hum Brain Mapp. 2012 Sep; 33(9)( doi: 10.1002/hbm.21354): p. 2211-23.

(36) Burghy C, Stodola D, Ruttle P, Molloy E, Armstrong J, Oler J, et al. Developmental pathways to amygdala-prefrontal function and internalizing symptoms in adolescence. Nat Neurosci. 2012 Dec; 15(12): p. 1736-41.

(37) Buchheim A, Erk S, George C, Kachele H, Ruchsow M, Spitzer M, et al. Measuring attachment representation in an FMRI environment: a pilot study. Psychopathology. 2006; 39(3): p. $144-52$.

(38) Lu S, Gao W, Wei Z, Wu W, Liao M, Ding Y, et al. Reduced cingulate gyrus volume associated with enhanced cortisol awakening response in young healthy adults reporting childhood trauma. PLoS One. 2013 Jul; 8(7):e69350(doi: 10.1371/journal.pone.0069350).

(39) Vaisvaser S, Lin T, Admon R, Podlipsky I, Greenman Y, N S, et al. Neural traces of stress: cortisol related sustained enhancement of amygdala-hippocampal functional connectivity. Front Hum Neurosci. 2013 Jul; 5;7:313(doi: 10.3389/fnhum.2013.00313. eCollection 2013).

(40) Quirin M, Gillath O, Pruessner J, Eggert L. Adult attachment insecurity and hippocampal cell density. Soc Cogn Affect Neurosci. 2010 Mar; 5(1): p. 39-47.

(41) Henry J. Psychological and physiological responses to stress: the right hemisphere and the hypothalamo-pituitary-adrenal axis, an inquiry into problems of human bonding. Acta Physiol Scand Suppl. 1997; 640: p. 10-25.

(42) Pierrehumbert B, Torrisi R, Glatz N, Dimitrova N, Heinrichs M, Halfon O. The influence of attachment on perceived stress and cortisol response to acute stress in women sexually abused in childhood or adolescence. Psychoneuroendocrinology. $2009 \mathrm{Jul} ; 34(6)$ : p. 924-38.

(43) Dienstbier R. Arousal and physiological toughness: implications for mental and physical health. Psychol Rev. 1989; 96: p. 84-100.

(44) Tops M, Riese H, Oldehinkel A, Rijsdijk F, Ormel J. Rejection sensitivity relates to hypocortisolism and depressed mood state in young women. Psychoneuroendocrinology. 2008 Jun; 33(5)(doi: 10.1016/j.psyneuen.2008.01.011): p. 551-9.

(45) Murray-Close D, Han G, Cicchetti D, Crick N, Rogoch F. Neuroendocrine Regulation and Physical and Relational Aggression: The Moderating Roles of Child Maltreatment and Gender. Dev Psychol. 2008; 44(4): p. 1160-1176.

(46) Henry J. Biological basis of the stress response. Integr Physiol Behav Sci. 1992 JanMar; 27(1): p. 66-83.

(47) Avitsur R, Stark J, Sheridan J. Social stress induces glucocorticoid resistance in subordinate animals. Horm Behav. 2001 Jun; 39(4): p. 247-57. 
ORIGINALES Y REVISIONES

(48) Monroy Cortés B, Palacios Cruz L. Resiliencia: ¿Es posible medirla e influir en ella? Salud Ment (Mexico City). 2011 May-Jun; 34(3): p. 237-46.

(49) Badanes L, Watamura S, Hankin B. Hypocortisolism as a potential marker of allostatic load in children: associations with family risk and internalizing disorders. Dev Psychopathol. 2011 Aug; 23(3)( doi: 10.1017/S095457941100037X): p. 881-96.

(50) Hellhammer J, Schlotz W, Stone A, Pirke K, Hellhammer D. Allostatic load, perceived stress, and health: a prospective study in two age groups. Ann N Y Acad Sci. 2004 Dec; 1032: p. 8-13.

(51) McVicar A, Ravalier J, Greenwood C. Biology of Stress Revisited: Intracellular Mechanisms and the Conceptualization of Stress. Stress Health. 2013 Jul; (doi: 10.1002/smi.2508).

(52) McEwen B, Biron C, Brunson K, Bulloch K, Chambers W, Dhabhar F, et al. The role of adrenocorticoids as modulators of immune function in health and disease: neural, endocrine and immune interactions. Brain Res Brain Res Rev. 1997 Feb; 23(1-2): p. 79-133.

(53) Karb R, Elliott M, Dowd J, Morenoff J. Neighborhood-level stressors, social support, and diurnal patterns of cortisol: the Chicago Community Adult Health Study. Soc Sci Med. 2012 Sep; 75(6)( doi: 10.1016/j.socscimed.2012.03.031): p. 1038-47.

(54) Silverman M, Sternberg E. Glucocorticoid regulation of inflammation and its functional correlates: from HPA axis to glucocorticoid receptor dysfunction. Ann N Y Acad Sci. 2012 Jul; 1261:55-63(doi: 10.1111/j.1749-6632.2012.06633.x).

(55) Faresjö Å, Theodorsson E, Chatziarzenis M, Sapouna V, Claesson H, Koppner J, et al. Higher perceived stress but lower cortisol levels found among young Greek adults living in a stressful social environment in comparison with Swedish young adults. PLoS One. 2013 Sep ; 16;8(9):e73828(doi: 10.1371/journal.pone.0073828).

(56) Fries E, Hesse J, Hellhammer J, Hellhammer D. A new view on hypocortisolism. Psychoneuroendocrinology. 2005; 1(7).

(57) Gustafsson P, Anckarsäter H, Lichtenstein P, Nelson N, Gustafsson P. Does quantity have a quality all its own? Cumulative adversity and up- and down-regulation of circadian salivary cortisol levels in healthy children. Psychoneuroendocrinology. 2010 Oct; 35(9)(doi: 10.1016/j.psyneuen.2010.04.004): p. 1410-5.

(58) Gaillard R. Interaction between the hypothalamo-pituitary-adrenal axis and the immunological system. Ann Endocrinol. 2001; 62(2): p. 155-163.

(59) Petrelluzzi K, Garcia M, Petta C, Grassi-Kassisse D, Spadari-Bratfisch R. Salivary cortisol concentrations, stress and quality of life in women with endometriosis and chronic pelvic pain. Stress. 2008 Sep; 11(5)( doi: 10.1080/10253890701840610): p. 390-7.

(60) Sarker M, Franks S, Caffrey J. Direction of post-prandial ghrelin response associated with cortisol response, perceived stress and anxiety, and self-reported coping and hunger in obese women. Behav Brain Res. 2013 Nov; 15;257(doi: 10.1016/j.bbr.2013.09.046): p. 197-200.

(61) Geliebter A, Carnell S, Gluck M. Cortisol and ghrelin concentrations following a cold pressor stress test in overweight individuals with and without night eating. Int J Obes (Lond). 2013 Aug; 37(8)(doi: 10.1038/ijo.2012.166): p. 1104-8.

(62) Kidd T, Hamer M, Steptoe A. Examining the association between adult attachment style and cortisol responses to acute stress. Psychoneuroendocrinology. 2011; 36: p. 771-779.

(63) Simeon D, Yehuda R, Cunill R, Knutelska M, Putnam F, Smith L. Factors associated with resilience in healthy adults. Psychoneuroendocrinology. 2007 Sep-Nov; 32(8-10): p. 1149-52. 
(64) Nolte T, Guiney J, Fonagy P, Mayes L, Luyten P. Interpersonal stress regulation and the development of anxiety disorders: an attachment-based developmental framework. Front Behav Neurosci. 2011 Sep.

(65) Hruby R, Hasto J, Minarik P. Attachment in integrative neuroscientific perspective. Neuro Endocrinol Lett. 2011; 32(2): p. 111-20.

(66) González-Torres M. Psychoanalysis and neurosciences. Friends or enemies? In International Forum of Psychoanalysis; 8 Sept 2011.

(67) Gervai J. Environmental and genetic influences on early attachment. Child Adolesc Psychiatry Ment Health. 2009; 3:25.

(68) Insel T, Young L. The neurobiology of attachment. Nat Rev Neurosci. 2001; 2, Feb.

(69) Doom J, Gunnar M. Stress physiology and developmental psychopathology: Past, present, and future. Dev Psychopathol. 2013 Nov; 25(4 Pt 2)(doi: 10.1017/S0954579413000667): p. 1359-73.

(70) Mommersteeg P, Keijsers G, Heijnen C, Verbraak M, van Doornen L. Cortisol deviations in people with burnout before and after psychotherapy: a pilot study. Health Psychol. 2006 Mar; 25(2): p. 243-8.

(71) Olff M, Güzelcan Y, de Vries G, Assies J, Gersons B. HPA- and HPT-axis alterations in chronic posttraumatic stress disorder. Psychoneuroendocrinology. 2006 Nov; 31(10): p. 1220-30.

(72) Steudte S, Kirschbaum C, Gao W, Alexander N, Schönfeld S, Hoyer J, et al. Hair cortisol as a biomarker of traumatization in healthy individuals and posttraumatic stress disorder patients. Biol Psychiatry. 2013 Nov; 1;74(9)(doi: 10.1016/j.biopsych.2013.03.011): p. 639-46.

(73) Powell D, Liossi C, Moss-Morris R, Schlotz W. Unstimulated cortisol secretory activity in everyday life and its relationship with fatigue and chronic fatigue syndrome: a systematic review and subset meta-analysis. Psychoneuroendocrinology. 2013 Nov; 38(11)(doi: 10.1016/j.psyneuen.2013.07.004): p. 2405-22.

(74) Bicanic I, Postma R, Sinnema G, De Roos C, Olff M, Van Wesel F, et al. Salivary cortisol and dehydroepiandrosterone sulfate in adolescent rape victims with post traumatic stress disorder. Psychoneuroendocrinology. 2013 Mar; 38(3)(doi: 10.1016/j.psyneuen.2012.06.015): p. 408-15.

(75) Kakiashvili T, Leszek J, Rutkowski K. The medical perspective on burnout. Int J Occup Med Environ Health. 2013 Jun; 26(3)(doi: 10.2478/s13382-013-0093-3): p. 401-12.

(76) Petrowski K, Wintermann G, Schaarschmidt M, Bornstein S, Kirschbaum C. Blunted salivary and plasma cortisol response in patients with panic disorder under psychosocial stress. Int $\mathbf{J}$ Psychophysiol. 2013 Apr; 88(1)(doi: 10.1016/j.ijpsycho.2013.01.002): p. 35-9.

(77) Michopoulos V, Mancini F, Loucks T, Berga S. Neuroendocrine recovery initiated by cognitive behavioral therapy in women with functional hypothalamic amenorrhea: a randomized, controlled trial. Fertil Steril. 2013 Jun; 99(7)(doi: 10.1016/j.fertnstert.2013.02.036): p. 2084-91.

(78) Myers B, Greenwood-Van Meerveld B. Differential involvement of amygdala corticosteroid receptors in visceral hyperalgesia following acute or repeated stress. Am J Physiol Gastrointest Liver Physiol. 2012 Jan; 15;302(2)( doi: 10.1152/ajpgi.00353.2011): p. 260-6.

(79) Tak L, Rosmalen J. Dysfunction of stress responsive systems as a risk factor for functional somatic syndromes. J Psychosom Res. 2010 May; 68(5)(doi: 10.1016/j.jpsychores.2009.12.004): p. 461-8.

(80) Loevinger B, Shirtcliff E, Muller D, Alonso C, Coe C. Delineating psychological and biomedical profiles in a heterogeneous fibromyalgia population using cluster analysis. Clin Rheumatol. 2012 Apr; 31(4)( doi: 10.1007/s10067-011-1912-1): p. 677-85. 
(81) Kuehl L, Michaux G, Richter S, Schächinger H, Anton F. Increased basal mechanical pain sensitivity but decreased perceptual wind-up in a human model of relative hypocortisolism. Pain. 2010 Jun; 149(3)(doi: 10.1016/j.pain.2010.03.026): p. 539-46.

(82) Roberts A, Charler M, Papadopoulos A, Wessely S, Chalder T, Cleare A. Does hypocortisolism predict a poor response to cognitive behavioural therapy in chronic fatigue syndrome? Psychol Med. 2010 Mar; 40(3)(doi: 10.1017/S0033291709990390): p. 515-22.

(83) Ostrander M, Ulrich-Lai Y, Choi D, Richtand N, Herman J. Hypoactivity of the Hypothalamo-Pituitary-Adrenocortical Axis during Recovery from Chronic Variable Stress. Endocrinology. ; 147(4): p. 2008-2017.

(84) Parker K, Buckmaster C, Schatzberg A, Lyons D. Prospective investigation of stress inoculation in young monkeys. Arch Gen Psychiatry. 2004 Sep; 61(9): p. 933-941.

(85) Valros A, Munsterhjelm C, Puolanne E, Ruusunen M, Heinonen M, Peltoniemi O, et al. Physiological indicators of stress and meat and carcass characteristics in tail bitten slaughter pigs. Acta Vet Scand. 2013 Oct; 55(1)(75).

(86) Armario A, Vallès A, Dal-Zotto S, Márquez C, Belda X. A single exposure to severe stressors causes long-term desensitization of the physiological response to the homotypic stressor. Ann N Y Acad Sci. 2004 Sep; 7(3): p. 157-72.

(87) Hatzenbuehler M, McLaughlin K. Structural Stigma and Hypothalamic-Pituitary-Adrenocortical Axis Reactivity in Lesbian, Gay, and Bisexual Young Adults. Ann Behav Med. 2013 Oct 24.

(88) Miller G, Iverson K, Kemmelmeier M, Maclane C, Pistorello J, Fruzzetti A, et al. A Pilot Study of Psychotherapist Trainees' Alpha-Amylase and Cortisol Levels During Treatment of Recently Suicidal Clients With Borderline Traits. Prof Psychol Res Pr. 2010 Jun; 41(3): p. 228-35.

(89) Wang S. Traumatic stress and attachment. Acta Physiol Scand Suppl. 1997; 640: p. 164-9.

(90) Klaassens E, van Noorden M, Giltay E, van Pelt J, van Veen T, Zitman F. Effects of childhood trauma on HPA-axis reactivity in women free of lifetime psychopathology. Prog Neuropsychopharmacol Biol Psychiatry. 2009 Aug; 33(5): p. 889-94.

(91) Engert V, Efanov S, Dedovic K, Duchesne A, Dagher A, Pruessner J. Perceived earlylife maternal care and the cortisol response to repeated psychosocial stress. J Psychiatry Neurosci. 2010; 35(6): p. 370-7.

(92) Elzinga B, Roelofs K, Tollenaar M, Bakvis P, van Pelt J, Spinhoven P. Diminished cortisol responses to psychosocial stress associated with lifetime adverse events a study among healthy young subjects. Psychoneuroendocrinology. 2008 Feb; 33(2): p. 227-37.

(93) Lucas-Thompson R, Hostinar C. Family income and appraisals of parental conflict as predictors of psychological adjustment and diurnal cortisol in emerging adulthood. J Fam Psychol. 2013 Oct; 27(5)(doi: 10.1037/a0034373.): p. 784-94.

(94) Schmid B, Buchmann A, Trautmann-Villalba P, Blomeyer D, Zimmermann U, Schmidt $\mathrm{M}$, et al. Maternal stimulation in infancy predicts hypothalamic-pituitary-adrenal axis reactivity in young men. J Neural Transm. 2013 Aug; 120(8)( doi: 10.1007/s00702-013-0970-8): p. 1247-57.

(95) Luecken L. Childhood Attachment and Loss Experiences Affect Adult Cardiovascular and Cortisol Function. Psychosom Med. 1998; 60: p. 765-777.

(96) Bloch M, Peleg I, Koren D, Aner H, Klein E. Long-term effects of early parental loss due to divorce on the HPA axis. Horm Behav. Apr; 51(4): p. 516-23.

(97) Carpenter L, Tyrka A, Ross N, Khoury L, Anderson G, Price L. Effect of Childhood Emotional Abuse and Age on Cortisol Responsivity in Adulthood. Biol Psychiatry. 2009 Jul; 66(1): p. 69-75. 
(98) Brand S, Holsboer-Trachsler E, Naranjo J, Schmidt S. Influence of mindfulness practice on cortisol and sleep in long-term and short-term meditators. Neuropsychobiology. 2012; 65(3): p. 109-18.

(99) Marcus M, Fine P, Moeller F, Khan M, Pitts K, Swank P. Change in Stress Levels Following Mindfulness-based Stress Reduction in a Therapeutic Community. Addict Disord Their Treat. 2003; 2: p. 63-8.

(100) Turakitwanakan W, Mekseepralard C, Busarakumtragul P. Effects of mindfulness meditation on serum cortisol of medical students. J Med Assoc Thai. 2013 Jan; 96(Suppl 1:S90-5).

(101) Kotsou I, Nelis D, Grégoire J, Mikolajczak M. Emotional plasticity: conditions and effects of improving emotional competence in adulthood. J Appl Psychol. 2011 Jul; 96(4): p. 827-39.

(102) Zengin S, Kabul S, Al B, Sarcan E, Doğan M, Yildirim C. Effects of music therapy on pain and anxiety in patients undergoing port catheter placement procedure. Complement Ther Med. 2013 Dec; 21(6)(doi: 10.1016/j.ctim.2013.08.017): p. 689-96.

(103) Nakayama H, Kikuta F, Takeda H. A pilot study on effectiveness of music therapy in hospice in Japan. J Music Ther. 2009 Summer; 46(2): p. 160-72.

(104) MacLean C, Walton K, Wenneberg S, Levitsky D, Mandarino J, Waziri R, et al. Effects of the Transcendental Meditation program on adaptive mechanisms: changes in hormone levels and responses to stress after 4 months of practice. Psychoneuroendocrinology. 1997 May; 22(4): p. 277-95.

(105) Yadav R, Magan D, Mehta N, Sharma R, Mahapatra S. Efficacy of a short-term yogabased lifestyle intervention in reducing stress and inflammation: preliminary results. J Altern Complement Med. 2012 Jul; 18(7)( doi: 10.1089/acm.2011.0265): p. 662-7.

(106) Schut C, Weik U, Tews N, Gieler U, Deinzer R, Kupfer J. Psychophysiological effects of stress management in patients with atopic dermatitis: a randomized controlled trial. Acta Derm Venereol. 2013 Jan; 93(1)( doi: 10.2340/00015555-1415): p. 57-61.

(107) Dewitte M, De Houwer J, Goubert L, Buysse A. A multi-modal approach to the study of attachment-related distress. Biol Psychol. 2010 Sep; 85(1)( doi: 10.1016/j.biopsycho.2010.06.006): p. 149-62.

(108) Buchheim A, Labek K, Walter S, Viviani R. A clinical case study of a psychoanalytic psychotherapy monitored with functional neuroimaging. Front Hum Neurosci. 2013 Oct; 7:677(doi: 10.3389/fnhum.2013.00677).

(109) Streeter C, Gerbarg P, Saper R, Ciraulo D, Brown R. Effects of yoga on the autonomic nervous system, gamma-aminobutyric-acid, and allostasis in epilepsy, depression, and post-traumatic stress disorder. Med Hypotheses. 2012 May; 78(5)(doi: 10.1016/j.mehy.2012.01.021): p. 571-9.

(110) Matousek R, Pruessner J, Dobkin P. Changes in the cortisol awakening response (CAR) following participation in mindfulness-based stress reduction in women who completed treatment for breast cancer. Complement Ther Clin Pract. 2011 May; 17(2): p. 65-70.

(111) Carlson L, Doll R, Stephen J, Faris P, Tamagawa R, Drysdale E, et al. Randomized controlled trial of Mindfulness-based cancer recovery versus supportive expressive group therapy for distressed survivors of breast cancer. J Clin Oncol. 2013 Sep; 1;31(25)(doi: 10.1200/JCO.2012.47.5210): p. 3119-26.

(112) Hsiao F, Jow G, Kuo W, Chang K, Liu Y, Ho R, et al. The effects of psychotherapy on psychological well-being and diurnal cortisol patterns in breast cancer survivors. Psychother Psychosom. 2012; 81(3)(doi: 10.1159/000329178): p. 173-82.

(113) Cruess D, Antoni M, McGregor B, Kilbourn K, Boyers A, Alferi S, et al. Cognitivebehavioral stress management reduces serum cortisol by enhancing benefit finding among women being treated for early stage breast cancer. Psychosom Med. 2000 May; 62(3): p. 304-8. 
(114) Olff M, de Vries G, Güzelcan Y, Assies J, Gersons B. Changes in cortisol and DHEA plasma levels after psychotherapy for PTSD. Psychoneuroendocrinology. 2007 Jul; 32(6): p. 619-26.

(115) Roberts A, Papadopoulos A, Wessely S, Chalder T, Cleare A. Salivary cortisol output before and after cognitive behavioural therapy for chronic fatigue syndrome. J Affect Disord. 2009 May; 115(1-2)( doi: 10.1016/j.jad.2008.09.013): p. 280-6.

(116) Miller M, McKinney A, Kanter F, Korte K, Lovallo W. Hydrocortisone Suppression of the Fear-potentiated Startle Response and Posttraumatic Stress Disorder. Psychoneuroendocrinology. 2011 Aug; 36(7): p. 970-980.

(117) de Kleine R, Rothbaum B, van Minnen A. Pharmacological enhancement of exposurebased treatment in PTSD: a qualitative review. Eur J Psychotraumatol. 2013 Oct; 17;4( doi: 10.3402/ ejpt.v4i0.21626).

(118) Golier J, Caramanica K, Demaria R, Yehuda R. A Pilot Study of Mifepristone in Combat-Related PTSD. Depress Res Treat. 2012; 2012:393251(doi: 10.1155/2012/393251).

(119) Delahanty D, Gabert-Quillen C, Ostrowski S, Nugent N, Fischer B, Morris A, et al. The efficacy of initial hydrocortisone administration at preventing posttraumatic distress in adult trauma patients: a randomized trial. CNS Spectr. 2013 Apr; 18(2)(doi: 10.1017/S1092852913000096): p. 103-11.

(120) Zohar J, Yahalom H, Kozlovsky N, Cwikel-Hamzany S, Matar M, Kaplan Z, et al. High dose hydrocortisone immediately after trauma may alter the trajectory of PTSD: interplay between clinical and animal studies. Eur Neuropsychopharmacol. 2011 Nov; 21(11)(doi: 10.1016/j.euroneuro.2011.06.001): p. 796-809.

(121) Cleare A, Miell J, Heap E, Sookdeo S, Young L, Malhi G, et al. Hypothalamo-pituitaryadrenal axis dysfunction in chronic fatigue syndrome, and the effects of low-dose hydrocortisone therapy. J Clin Endocrinol Metab. 2001 Aug; 86(8): p. 3545-54.

(122) Cleare A, Heap E, Malhi G, Wessely S, O'Keane V, Miell J. Low-dose hydrocortisone in chronic fatigue syndrome: a randomised crossover trial. Lancet. 1999 Feb; 6;353((9151)): p. 455-8.

(123) Holtorf K. Diagnosis and Treatment of Hypothalamic-Pituitary-Adrenal (HPA) Axis Dysfunction in Patients with Chronic Fatigue Syndrome (CFS) and Fibromyalgia (FM). J. Chronic Fatigue Syndr. 2008; 14:3(doi:10.1300/J092v14no3_06).

(124) Ben-Zvi A, Vernon S, Broderick G. Model-Based Therapeutic Correction of Hypothalamic Pituitary-Adrenal Axis Dysfunction. PLoS Comput Biol. 2009 Jan; 5(1) :e1000273.(doi: 10.1371/journal.pcbi.1000273).

(125) Cicchetti D, Rogosch F. Personality, adrenal steroid hormones, and resilience in maltreated children: A multi-level perspective. Dev Psychopathol. 2007; 19(3): p. 787-809.

(126) Karatsoreos I, McEwen B. Psychobiological allostasis: resistance, resilience and vulnerability. Trends Cogn Sci. 2011 Dec; 15(12)(doi: 10.1016/j.tics.2011.10.005): p. 576-84.

(127) Hoge E, Austin E, Pollack M. Resilience, research evidence and conceptual considerations for posttraumatic stress disorder. Depress Anxiety. 2007; 24(2): p. 139-152.

(128) Earvolino-Ramirez M. Resilience: a concept analysis. Nurs Forum. 2007 Apr; 42(2): p. 73-82.

(129) Simeon D, Yehuda R, Cunill R, Knutelska M, Putnam F, Smith L. Factors associated with resilience in healthy adults. Psychoneuroendocrinology. 2007 Sep; 32(8-10): p. 1149-1152.

(130) Ortigo K, Westen D, Defife J, Bradley B. Attachment, social cognition, and posttraumatic stress symptoms in a traumatized, urban population: evidence for the mediating role of object relations. J Trauma Stress. 2013 Jun; 26(3)(doi: 10.1002/jts.21815): p. 361-8. 
(131) Escolas S, Arata-Maiers R, Hildebrandt E, Maiers A, Mason S, Baker M. The impact of attachment style on posttraumatic stress disorder symptoms in postdeployed service members. US Army Med Dep J. 2012 Jul-Sep;: p. 54-61.

(132) Tosone C, Bettmann J, Minami T, Jasperson R. New York City social workers after 9/11: their attachment, resiliency, and compassion fatigue. Int J Emerg Ment Health. 2010 Spring; 12(2): p. 103-16.

(133) Granger D, Kivlighan K. Integrating biological, behavioral, and social levels of analysis in early child development. Child Dev. 2003 Jul; 74(4): p. 1058-63.

(134) Charney D. Psychobiological mechanisms of resilience and vulnerability. Am J Psychiatry. $2004 \mathrm{Feb}$; 161(2): p. 195-216.

(135) Haglund M, Nestadt P, Cooper N, Southwick S, Charney D. Psychobiological mechanisms of resilience, relevance to prevention and treatment of stress-related psychopathology. Dev Psychopathol. 2007; 19(3): p. 889-920.

(136) Rutten B, Hammels C, Geschwind N, Menne-Lothmann C, Pishva E, Schruers K, et al. Resilience in mental health: linking psychological and neurobiological perspectives. Acta Psychiatr Scand. 2013 Jul; 128(1)(doi: 10.1111/acps.12095): p. 3-20.

(137) Cherro M. La gran contribución de la teoría del apego a la Psiquiatría y a la Psicoterapia. Rev Psiquiatr Urug. Dic 2010; 74, no. 2: p. 123.

(138) Kähele H, Buchheim A, Schmücker G, Brisch K. Development, attachment and relationship: New psychoanalytical concepts. Contemporary Psychiatry. .

(139) Kochanska G. Emotional development in children with different attachment histories: the first three years. Child Dev. 2011 March/Apr; 72, no. 2: p. 474-490.

(140) van Bakel H, Riksen-Walraven J. Adrenocortical and behavioral attunement in parents with 1-year-old infants. Dev Psychobiol. 2008 Mar; 50(2)( doi: 10.1002/dev.20281): p. 196-201.

(141) Simpson J, Collins W, Tran S, Haydon K. Attachment and the experience and expression of emotions in romantic relationships: a developmental perspective. J Pers Soc Psychol. 2007; 92, no. 2 : p. 355-367.

(142) Ainsworth M. Attachments beyond infancy. Am Psychol. 1989; 44: p. 709-716.

(143) Lyons D, Parker K, Schatzberg A. Animal Models of Early Life Stress: Implications for Understanding Resilience. Dev Psychobiol. 2010 Jul; 52(5): p. 402-410.

(144) Kim P, Leckman J, Mayes L, Newman M, Feldman R, Swain J. Perceived quality of maternal care in childhood and structure and function of mothers' brain. Dev Sci. 2010 Jul; 13(4)(doi: 10.1111/j.1467-7687.2009.00923.x): p. 662-73.

(145) Siegel D. La mente en desarrollo. Cómo interactúan las relaciones y nuestro cerebro para modelar nuestro ser: Desclee De Brouwer; 2007.

(146) Retolaza A. ¿Induce a error el principio de causalidad? Norte de Salud Mental. 2011; IX(40): p. 72-89.

(147) Nietzsche F. (1886). In Más allá del Bien y del Mal. Madrid: Edaf; 1994.

(148) Rizzolatti G, Craighero L. The Mirror-Neuron System. Annu Rev Neurosci. 2004; 27 : p. 169-192.

(149) Damasio A. Descartes' Error: emotion, reason and the human brain London: Vintage; 1994.

(150) Le Doux J. The Emotional Brain Nueva York: Simon \& Schuster; 1996.

(151) Schore A. The effects of early relational trauma on right brain development, affect regulation, and infant mental health. Infant Ment Health J. 2011; 22: p. 201-269. 
ORIGINALES Y REVISIONES

(152) Lyons D, Parker K, Katz M, Schatzberg A. Developmental cascades linking stress inoculation, arousal regulation and resilience. Front Behav Neurosci. 2009 Sep; doi: 10.3389/neuro.08.032.2009.

(153) Fonagy P, Target M, Gergely G, Allen J, Bateman A. The Developmental Roots of Borderline Personality Disorder in Early Attachment Relationships. Psychoanalytical Inquiry. 2003; 23: p. 412-459. 